\title{
Comparative Assessment on Performance of Aseel and Kadaknath in Hot and Humid Conditions in Tropics
}

\author{
M. Shanmathy", J.S. Tyagi, M. Gopi, J. Mohan, P. Beulah and D. Ravi Kumar \\ ICAR-Central Avian Research Institute, Izatnagar-243 122(UP), India \\ *Corresponding author
}

\section{A B S T R A C T}

\begin{tabular}{|c|c|}
\hline & Native birds are considered for cross breeding to develop thermal tolerance and free range \\
\hline Keywords & $\begin{array}{l}\text { present study was conducted to assess the performance of two Indigenous breeds Aseel } \\
\text { and Kadaknath under hot and humid conditions. The two indigenous breeds were studied }\end{array}$ \\
\hline $\begin{array}{l}\text { Kadaknath, Aseel, } \\
\text { Hot-humid, Body } \\
\text { weight, Feed } \\
\text { efficiency }\end{array}$ & $\begin{array}{l}\text { for various performance parameters for a period of } 12 \text { weeks under standard } \\
\text { managemental conditions. Hatch weight was significant among the breeds and sex with } \\
\text { Aseel recording higher weight }(29.08 \text { vs. } 25.30) \text {. Significant }(\mathrm{P}<0.01) \text { difference for } \\
\text { weekly body weights and weekly body weight gain was observed between the two breeds. }\end{array}$ \\
\hline Article Info & During the overall experimental period, Aseel had higher $(\mathrm{P}<0.01)$ body weight than \\
\hline $\begin{array}{l}\text { Accepted: } \\
18 \text { April } 2018 \\
\text { Available Online: } \\
10 \text { May } 2018\end{array}$ & $\begin{array}{l}\text { Breed influence on feed intake was highly significant }(\mathrm{P}<0.01) \text { for the first } 8 \text { weeks with } \\
\text { Aseel recording higher feed intake. Feed intake for } 4,8 \text { and } 12^{\text {th }} \text { week in Aseel and } \\
\text { Kadaknath were } 133 \text { vs. } 95,381 \text { vs. } 172,504 \text { vs } 483 \mathrm{~g} / \mathrm{d} \text { respectively. FCR ranged between } \\
2.26 \text { to } 10.51 \text { for the birds during the experimental period. From the study it may be }\end{array}$ \\
\hline & $\begin{array}{l}\text { concluded that Aseel (males and females) had better production performance than the } \\
\text { Kadaknath which can be used for crossbreeding. }\end{array}$ \\
\hline
\end{tabular}

\section{Introduction}

Poultry has an essential role in terms of protein and mineral supplement in India. Recently native chickens are more popular for their unique characteristics. India is home to more than 20 breeds (Panda and Praharaj 2002). Among them Aseel and Kadaknath are becoming progressively more popular as pure and out-crossed lines for their benefits in production traits and resistance to disease (Arora et al., 2011; Haunshi et al., 2011). Aseel breed have its origin in Andhra Pradesh. These birds are renowned for its stamina, pugnacity, majestic gait and fighting qualities (Panda and Mahapatra. 1989). Despite of its unique qualities, the Aseel is under the threat of extinction because of its poor production performance (Mohan et al., 2008). Kadaknath is an important indigenous breed of poultry inhabiting vast areas of Western Madhya Pradesh mainly in Jhabua and Dhar Districts (Thakur et al., 2006). In all the three varieties of Kadaknath breed most of the internal organs exhibit intense black colouration which is due to the deposition of melanin pigment in the connective tissue of organs and in the dermis (Rao and Thomas, 1984). Although the 
Kadaknath has many unique characteristics, they are being neglected because of its poor production potential Haunshi et al., (2011). Even though indigenous or native stock show a poor performance relative to highly selected commercial lines, they have the ability to survive in challenging environments (Crawford and Christman, 1992). Despite a drastic increase in the import of high yielding strains from across the world, the local breed are still preferred in their native environment mainly due to its special capabilities i.e., good foraging, less cost and efficient mothers (Biswas et al., 2010). Performance potentials have to be documented in indigeneous breeds for better insight into the breeds. Endangered status of the breeds also makes it vital to document the breeds as extensive crossbreeding has also diluted the original genes. The aim of the present study was to evaluate and compare the performance traits between kadaknath and aseel.

\section{Materials and Methods}

\section{Ethical approval}

The study was carried out as per the guidelines and approval of Institute Animal Ethical Committee (IAEC) and Committee for the purpose of control and supervision of experiments on animals (CPCSEA).

\section{Experimental design and dietary treatments}

The Institute is located at an altitude of $169 \mathrm{~m}$ above the mean sea level, at $28^{\circ} 22^{\prime}$ latitude and $79.24^{\circ} \mathrm{E}$ longitude. The place experiences extreme hot and humid (approximately $45^{\circ} \mathrm{C}$ ) and cold (approximately $5^{\circ} \mathrm{C}$ ) and the $\mathrm{RH}$ ranges between $15 \%$ and $99 \%$. A total of 400 hatching eggs of each breed (Aseel peela and Kadaknath) were obtained from the Experimental Desi Farm, ICAR- Central Avian Research Institute, Izatnagar, India and were incubated at Experimental Hatchery Unit of the institute for 21 days. Day old chicks were sexed by Japanese method of vent sexing and were reared for a period of three months (27 July-8 September 2016).All the birds (Aseel 175 male and 118 female, Kadaknath male 130 and 134). All the birds were maintained under the standard managemental conditions with feed and water ad libitum. The growth period is divided into two phases, initial brooding phase (0-6 weeks) chicks were maintained in cages and growing phase where the birds were reared in both cage and litter till $12^{\text {th }}$ week of age to compare their performances. Birds were offered as per ICAR 2013 recommendations (Table 1).

\section{Production performance}

Day old chick weights for birds were recorded followed by weekly body weight till 12 weeks of age. Body weights were recorded on weekly basis and body weight gain was calculated. Feed intake was recorded and feed efficiency was calculated. Mortality was taken into the account while calculating FCR, weight gain and feed intake was adjusted to arrive at corrected FCR.

\section{Statistical analysis}

The data collected pertaining to various performance parameters were subjected to $2 \mathrm{X} 2 \mathrm{X} 2 \mathrm{X} 2$ factorial analysis to assess the interaction as well as the effect of breed. The analysis was carried out using SPSS V. 20.0 package, USA. The means were for significance by using Duncan's multiple range tests (Table 2).

\section{Results and Discussion}

\section{Weekly body weight}

The means of body weight (BW) of groups is given in Tables 3 and 4 . The four groups 
comprising male and female sexes of Aseel and Kadaknath under the present study showed significant difference $(\mathrm{P}<0.01)$ in weekly body weight under hot and humid condition. Aseel female recorded higher body weight for first 6 weeks. Day old weight of Aseel male and Aseel female in hot and humid were $29.34 \pm 0.36 \mathrm{~g}$ and $28.69 \pm 0.26 \mathrm{~g}$ which is lower than $33.18 \pm 0.91 \mathrm{~g}$ reported by Valavan et al., (2016). Day old weight of Kadaknath male and Kadaknath female in hot and humid were $25.29 \pm 0.23 \mathrm{~g}$ and $25.31 \pm 0.29 \mathrm{~g}$ which is lower than $28.55 \pm 0.12 \mathrm{~g}$ reported by Hanushi et al., (2011).

Pratap et al., (2014) reported $4^{\text {th }}$ week body weight of Kadaknath male and female as 144 and $128 \mathrm{~g}$ respectively. In the present study, Kadaknath male and female weighed $109.44 \pm 1.75$ and $115.52 \pm 1.81 \mathrm{~g}$ respectively which are in close agreement with the $4^{\text {th }}$ week body weight reported by Parmar (2003) as 105 g. Weights of Kadaknath male and female in first 6 weeks were closely comparable and post separation into cage and litter, Kadaknath male recorded higher weights than Kadaknath female. Aseel always had higher body weight in all the 12 weeks. Higher BW in Aseel may be due to taller stature attained in course of selection for fighting and natural tendency for robust muscle development. It may be due to higher tendency of muscle growth in males. Chatterjee (2007) reported 8 week body weights of Aseel and Kadaknath as $393 \mathrm{~g}$ and $275 \mathrm{~g}$ which is comparable to the observation of the present study of $392.09 \pm 4.16 \mathrm{~g}$ and $278.61 \pm 3.45$ g. Caged birds had higher body weight than birds on deep litter.

\section{Weekly weight gain}

Decreased growth performance in terms of average body weights, total body weight gain, total feed intake and feed efficiency in chickens due to negative influences of environmental stress has been explained by various workers (Siegel, 1995; Zulkifli et al., 2000; Mashaly et al., 2004). Weekly weight gain of groups differed significantly $(\mathrm{P}<0.01)$ (Table 5 and 6). Weight gain amongst groups was in range $15.16 \pm 0.48$ to $110.25 \pm 6.18 \mathrm{~g}$ in course of 12 weeks. The reason for lower weight gain in indigenous breeds from same breeding stock can be the non-selected origin of Aseel and Kadaknath.

The flock of Kadaknath maintained at CARI is random bred population with an aim to conserve the breed for posterity (Pratap et al., 2014). Significantly $(\mathrm{P}<0.05)$ higher weight gain was observed in Aseel than Kadaknath. Higher body weight of Aseel birds in relation to Kadaknath has also been reported by Haunshi et al., (2011).

Higher weight gain was observed in cage birds. Vecerek et al., (2002) reported effect of high environmental temperatures on metabolic changes were represented by reduced growth intensity and increased mortality of chickens during the fattening.

\section{Weekly feed intake}

Aseel male had highest feed intake for first 6 weeks except week 1 and week 4 when Aseel female recorded highest feed intake. Effect of breed on feed intake was significant $(\mathrm{P}<0.01)$ for first 6 weeks then significant $(\mathrm{P}<0.05)$ up to 11 weeks (Table 7 and 8). Aseel recorded higher feed intake than Kadaknath which can be explained by higher metabolic demand due to higher weight gain. Weekly feed intake (g) per bird in 1, 2, 3 month old Aseel and Kadaknath was $132.99 \pm 6.24,381.10 \pm 25.61$, $504.35 \pm 20.86$ and $94.58 \pm 1.37,172.00 \pm 9.56$, $482.65 \pm 32.34$ respectively. Gupta et al., (2000) studied feed efficiency of Aseel chicken and reported average weekly feed consumption per bird was 28.82, 123.89, $305.12,538.46$ and $646.15 \mathrm{~g}$ during $1,4,8,12$ and 16 weeks of age. 
Table.1 Ingredient composition of chick starter and grower ration on dry matter basis

\begin{tabular}{|c|c|c|}
\hline Ingredient (\%) & Chick starter (0-6 weeks) & Grower (7-12 weeks) \\
\hline Maize & 55 & 51 \\
\hline De-oiled rice bran & 10.59 & 28.91 \\
\hline Soybean meal (46\% CP) & 20 & 5.5 \\
\hline Guar Korma & 4 & 4 \\
\hline Rape Seed Meal & 3 & 4 \\
\hline Fish meal & 4 & 4 \\
\hline Marble chips & - & 1 \\
\hline Limestone & 1.105 & 0.6 \\
\hline Di-calcium Phosphate & 1.2 & 0.4 \\
\hline Salt & 0.25 & 0.25 \\
\hline DL- methionine & 0.04 & 0.02 \\
\hline L-Lysine & 0.04 & 0.02 \\
\hline Trace MineralPremix ${ }^{1}$ & 0.1 & 0.1 \\
\hline VitaminPremix $^{2}$ & 0.15 & 0.1 \\
\hline Vitamin B-complex ${ }^{3}$ & 0.015 & 0.01 \\
\hline Calcium Chloride & 0.05 & - \\
\hline Toxin binder & 0.05 & 0.05 \\
\hline Vitamin C & 0.01 & 0.04 \\
\hline Sodium Bicarbonate & 0.3 & - \\
\hline Liver tonic & 0.025 & - \\
\hline Geriforte & 0.025 & - \\
\hline Coccidiostat & 0.05 & 0.04 \\
\hline
\end{tabular}

${ }^{1}$ Trace mineral premix (mg/ kg diet) $\mathrm{Mg}-300, \mathrm{Mn}-55, \mathrm{I}-0.4, \mathrm{Fe}-56, \mathrm{Zn}-30$ and $\mathrm{Cu}-4.0$.

${ }^{2}$ Vitamin premix: Vitamin A - 8250 IU, Vitamin $\mathrm{D}_{3}-1200 \mathrm{IU}$, Vitamin K - $1 \mathrm{mg}$, Vitamin E - 40 (mg)

${ }^{3}$ Vitamin $B_{1}-2 \mathrm{mg}$, Vitamin $\mathrm{B}_{2}-4 \mathrm{mg}$, Vitamin $\mathrm{B}_{12}-10 \mathrm{mg}$. 
Table. 2 Influence of breed on body weight $(\mathrm{g})$ during brooder phase

\begin{tabular}{|c|c|c|c|c|c|c|c|c|}
\hline Breed & Sex & Hatch & 1 week & 2 week & 3 week & 4 week & 5 week & 6 week \\
\hline \multicolumn{9}{|c|}{ Interaction effect } \\
\hline \multirow[t]{2}{*}{ Aseel } & Females & $28.69^{\mathrm{a}} \pm 0.26$ & $44.07^{\mathrm{a}} \pm 0.52$ & $67.62^{\mathrm{a}} \pm 1.03$ & $99.45^{\mathrm{a}} \pm 1.55$ & $138.67^{\mathrm{a}} \pm 2.34$ & $190.88^{\mathrm{a}} \pm 2.81$ & $263.96^{\mathrm{a}} \pm 3.97$ \\
\hline & Males & $29.34^{\mathrm{a}} \pm 0.36$ & $43.88^{\mathrm{a}} \pm 0.50$ & $63.26^{\mathrm{b}} \pm 0.82$ & $94.52^{\mathrm{a}} \pm 1.29$ & $128.75^{\mathrm{a}} \pm 2.00$ & $184.05^{\mathrm{a}} \pm 2.74$ & $259.79^{\mathrm{a}} \pm 3.92$ \\
\hline \multirow[t]{2}{*}{ Kadaknath } & Females & $25.31^{b} \pm 0.29$ & $40.14^{\mathrm{b}} \pm 0.48$ & $58.09^{c} \pm 0.76$ & $85.24^{c} \pm 1.25$ & $115.52^{c} \pm 1.81$ & $146.09^{b} \pm 2.49$ & $186.00^{\mathrm{b}} \pm 3.15$ \\
\hline & Males & $25.29^{b} \pm 0.23$ & $38.74^{\mathrm{c}} \pm 0.41$ & $57.69^{c} \pm 0.85$ & $82.60^{c} \pm 1.25$ & $109.44^{\mathrm{d}} \pm 1.75$ & $140.41^{b} \pm 2.64$ & $185.47^{\mathrm{b}} \pm 3.21$ \\
\hline \multicolumn{2}{|l|}{ P-value } & 0.000 & 0.000 & 0.000 & 0.000 & 0.000 & 0.000 & 0.000 \\
\hline \multicolumn{9}{|l|}{ Breed effect } \\
\hline \multicolumn{2}{|l|}{ Aseel } & $29.08^{\mathrm{a}}+0.24$ & $43.96^{\mathrm{a}} \pm 0.36$ & $65.06^{\mathrm{a}} \pm 0.66$ & $96.58^{\mathrm{a}} \pm 1.00$ & $132.93^{\mathrm{a}} \pm 1.55$ & $186.98^{\mathrm{a}} \pm 1.98$ & $261.59^{\mathrm{a}} \pm 2.81$ \\
\hline \multicolumn{2}{|l|}{ Kadaknath } & $25.30^{\mathrm{b}}+0.18$ & $39.47^{\mathrm{b}} \pm 0.32$ & $57.90^{b} \pm 0.57$ & $83.98^{b} \pm 0.89$ & $112.63^{b} \pm 1.28$ & $143.40^{b} \pm 1.82$ & $185.75^{\mathrm{b}} \pm 2.25$ \\
\hline \multicolumn{2}{|l|}{ P-value } & 0.000 & 0.000 & 0.000 & 0.000 & 0.000 & 0.000 & 0.000 \\
\hline
\end{tabular}

Means within columns bearing different superscripts differ significantly $(\mathrm{P}<0.05)$

Table.3 Influence of breed on body weight $(\mathrm{g})$ during grower phase

\begin{tabular}{|c|c|c|c|c|c|c|c|c|}
\hline Breed & Sex & Rearing & 7week & 8 week & 9 week & 10week & 11week & 12week \\
\hline \multicolumn{9}{|c|}{ Interaction effect } \\
\hline \multirow[t]{4}{*}{ Aseel } & \multirow[t]{2}{*}{ Females } & Cage & $354.69^{\mathrm{a}} \pm 6.38$ & $489.83^{\mathrm{a}} \pm 9.49$ & $339.63^{\mathrm{a}} \pm 3.75$ & $566.69^{\mathrm{a}} \pm 11.87$ & $632.54^{\mathrm{a}} \pm 12.76$ & $712.56^{\mathrm{a}} \pm 13.50$ \\
\hline & & Litter & $336.87^{\mathrm{ab}} \pm 6.36$ & $429.45^{\mathrm{b}} \pm 8.13$ & $489.83^{b} \pm 9.49$ & $480.85^{c} \pm 9.11$ & $530.97^{\mathrm{c}} \pm 10.04$ & $588.47^{b} \pm 10.83$ \\
\hline & \multirow[t]{2}{*}{ Males } & Cage & $330.52^{\mathrm{b}} \pm 9.50$ & $443.15^{\mathrm{b}} \pm 11.75$ & $429.45^{\mathrm{b}} \pm 8.13$ & $521.47^{b} \pm 12.50$ & $576.10^{b} \pm 13.46$ & $684.77^{\mathrm{a}} \pm 14.81$ \\
\hline & & Litter & $350.63^{\mathrm{ab}} \pm 6.31$ & $447.28^{\mathrm{b}} \pm 7.63$ & $443.15^{\mathrm{b}} \pm 11.75$ & $504.09^{b c} \pm 8.42$ & $547.40^{c} \pm 8.25$ & $594.35^{\mathrm{b}} \pm 8.90$ \\
\hline \multirow[t]{4}{*}{ Kadaknath } & \multirow[t]{2}{*}{ Females } & Cage & $240.51^{\mathrm{d}} \pm 7.08$ & $297.81^{\mathrm{d}} \pm 7.37$ & $447.28^{\mathrm{d}} \pm 7.63$ & $343.00 \mathrm{~d}^{\mathrm{e}} \pm 7.78$ & $391.55^{\mathrm{d}} \pm 8.30$ & $446.79^{c} \pm 8.95$ \\
\hline & & Litter & $251.55^{\mathrm{d}} \pm 5.14$ & $292.93^{\mathrm{d}} \pm 5.83$ & $297.81^{\mathrm{d}} \pm 7.37$ & $326.06^{\mathrm{e}} \pm 6.41$ & $358.86^{\mathrm{e}} \pm 7.45$ & $400.77^{\mathrm{e}} \pm 8.79$ \\
\hline & \multirow[t]{2}{*}{ Males } & Cage & $255.58^{\mathrm{d}} \pm 7.74$ & $322.67^{\mathrm{cd}} \pm 9.03$ & $292.93^{c} \pm 5.83$ & $364.09^{\mathrm{d}} \pm 10.40$ & $414.84^{\mathrm{d}} \pm 11.44$ & $462.24^{c} \pm 12.12$ \\
\hline & & Litter & $277.85^{\mathrm{c}} \pm 6.79$ & $325.82^{c} \pm 7.76$ & $322.67^{c} \pm 9.03$ & $362.74^{\mathrm{d}} \pm 8.09$ & $399.21^{\mathrm{d}} \pm 8.20$ & $451.21^{\mathrm{c}} \pm 9.12$ \\
\hline \multicolumn{3}{|l|}{ P-value } & 0.000 & 0.000 & 0.000 & 0.000 & 0.000 & 0.000 \\
\hline \multicolumn{9}{|l|}{ Breed effect } \\
\hline \multicolumn{3}{|l|}{ Aseel } & $343.73^{\mathrm{a}} \pm 3.63$ & $392.09^{\mathrm{a}} \pm 4.16$ & $450.88^{\mathrm{a}} \pm 4.73$ & $515.34^{\mathrm{a}} \pm 5.42$ & $567.41^{\mathrm{a}} \pm 5.82$ & $637.99^{\mathrm{a}} \pm 6.71$ \\
\hline \multicolumn{3}{|l|}{ Kadaknath } & $256.96^{\mathrm{b}} \pm 3.40$ & $278.61^{b} \pm 3.45$ & $308.95^{\mathrm{b}} \pm 3.80$ & $347.67^{b} \pm 4.12$ & $388.70^{\mathrm{b}} \pm 4.52$ & $437.58^{\mathrm{b}} \pm 5.04$ \\
\hline \multicolumn{3}{|l|}{ P-value } & 0.000 & 0.000 & 0.000 & 0.000 & 0.000 & 0.000 \\
\hline
\end{tabular}


Table.4 Influence of breed on body weight gain (g) during brooder phase

\begin{tabular}{|c|c|c|c|c|c|c|c|}
\hline Breed & Sex & 1 week & 2 week & 3 week & 4 week & 5 week & 6 week \\
\hline \multicolumn{8}{|c|}{ Interaction effect } \\
\hline \multirow[t]{2}{*}{ Aseel } & Females & $15.48^{\mathrm{a}} \pm 0.43$ & $24.04^{\mathrm{a}} \pm 0.77$ & $31.97^{\mathrm{a}} \pm 0.91$ & $39.29^{\mathrm{a}} \pm 1.24$ & $52.43^{\mathrm{a}} \pm 1.49$ & $72.97^{\mathrm{a}} \pm 2.45$ \\
\hline & Males & $15.16^{\mathrm{a}} \pm 0.48$ & $19.32^{\mathrm{b}} \pm 0.87$ & $32.08^{\mathrm{a}} \pm 1.36$ & $34.94^{\mathrm{b}} \pm 1.09$ & $54.95^{\mathrm{a}} \pm 1.31$ & $74.45^{\mathrm{a}} \pm 1.82$ \\
\hline \multirow[t]{2}{*}{ Kadaknath } & Females & $15.29^{\mathrm{a}} \pm 0.52$ & $18.00^{\mathrm{b}} \pm 0.62$ & $26.85^{b} \pm 0.92$ & $29.62^{c} \pm 1.20$ & $31.17^{\mathrm{b}} \pm 1.63$ & $38.19^{\mathrm{c}} \pm 2.51$ \\
\hline & Males & $13.54^{\mathrm{b}} \pm 0.42$ & $18.92^{\mathrm{b}} \pm 0.72$ & $25.02^{\mathrm{b}} \pm 0.92$ & $27.47^{\mathrm{c}} \pm 1.21$ & $31.40^{\mathrm{b}} \pm 1.44$ & $44.81^{\mathrm{b}} \pm 1.55$ \\
\hline P-value & & 0.000 & 0.022 & 0.000 & 0.000 & 0.000 & 0.000 \\
\hline \multicolumn{8}{|l|}{ Breed effect } \\
\hline Aseel & & $15.29^{\mathrm{a}} \pm 0.33$ & $21.28^{\mathrm{a}} \pm 0.61$ & $32.03^{\mathrm{a}} \pm 0.87$ & $36.77^{\mathrm{a}} \pm 0.83$ & $53.87^{\mathrm{a}} \pm 0.98$ & $73.81^{\mathrm{a}} \pm 1.47$ \\
\hline Kadaknath & & $14.45^{b} \pm 0.34$ & $18.44^{\mathrm{b}} \pm 0.47$ & $25.98^{\mathrm{b}} \pm 0.65$ & $28.60^{b} \pm 0.86$ & $31.27^{\mathrm{b}} \pm 1.09$ & $41.35^{\mathrm{b}} \pm 1.54$ \\
\hline P-value & & 0.000 & 0.000 & 0.000 & 0.000 & 0.000 & 0.000 \\
\hline
\end{tabular}

Means within columns bearing different superscripts differ significantly $(\mathrm{p}<0.05)$.

Table.5 Influence of breed on body weight gain (g) during grower phase

\begin{tabular}{|c|c|c|c|c|c|c|c|c|}
\hline Breed & Sex & Rearing & 7week & 8 week & 9 week & 10week & 11 week & 12week \\
\hline \multicolumn{9}{|c|}{ Interaction effect } \\
\hline \multirow[t]{4}{*}{ Aseel } & \multirow[t]{2}{*}{ Females } & Cage & $56.50^{\mathrm{a}} \pm 3.94$ & $63.85^{\mathrm{a}} \pm 2.21$ & $73.15^{\mathrm{a}} \pm 3.84$ & $78.69^{\mathrm{a}} \pm 4.01$ & $63.22^{a} \pm 3.00$ & $78.54^{b} \pm 4.44$ \\
\hline & & Litter & $47.19^{b} \pm 2.62$ & $43.87^{\mathrm{c}} \pm 2.21$ & $52.90^{\mathrm{c}} \pm 2.30$ & $51.40^{\mathrm{b}} \pm 2.60$ & $50.95^{\mathrm{bc}} \pm 1.99$ & $62.66^{c} \pm 3.84$ \\
\hline & \multirow[t]{2}{*}{ Males } & Cage & $52.59^{\mathrm{ab}} \pm 4.28$ & $52.61^{\mathrm{b}} \pm 3.87$ & $64.17^{\mathrm{b}} \pm 3.18$ & $79.93^{\mathrm{a}} \pm 4.59$ & $56.36^{\mathrm{ab}} \pm 4.35$ & $110.25^{\mathrm{a}} \pm 6.18$ \\
\hline & & Litter & $51.27^{\mathrm{ab}} \pm 2.40$ & $41.97^{c} \pm 2.07$ & $54.30^{\mathrm{c}} \pm 2.34$ & $53.34^{b} \pm 2.46$ & $43.90^{c} \pm 2.03$ & $51.43^{\mathrm{d}} \pm 2.78$ \\
\hline \multirow[t]{4}{*}{ Kadaknath } & \multirow[t]{2}{*}{ Females } & Cage & $38.55^{c} \pm 3.12$ & $21.75^{\mathrm{d}} \pm 1.85$ & $35.00^{\mathrm{d}} \pm 2.63$ & $45.98^{b c} \pm 2.00$ & $47.53^{\mathrm{c}} \pm 2.36$ & $52.57^{\mathrm{cd}} \pm 3.37$ \\
\hline & & Litter & $37.22^{c} \pm 2.41$ & $20.90^{\mathrm{d}} \pm 2.35$ & $21.84^{\mathrm{e}} \pm 2.01$ & $34.01 \mathrm{e} \pm 2.17$ & $32.54^{\mathrm{d}} \pm 2.39$ & $38.99^{e} \pm 2.86$ \\
\hline & \multirow[t]{2}{*}{ Males } & Cage & $33.21^{c} \pm 2.60$ & $24.53^{\mathrm{d}} \pm 2.45$ & $42.02^{\mathrm{d}} \pm 2.56$ & $40.76^{\mathrm{cd}} \pm 3.61$ & $49.93^{b c} \pm 3.25$ & $48.22^{\mathrm{de}} \pm 3.51$ \\
\hline & & Litter & $34.62^{c} \pm 1.92$ & $22.55^{\mathrm{d}} \pm 2.40$ & $28.18^{\mathrm{e}} \pm 2.02$ & $35.40^{\mathrm{e}} \pm 2.24$ & $31.36^{\mathrm{d}} \pm 2.10$ & $51.16^{\mathrm{d}} \pm 2.28$ \\
\hline \multicolumn{3}{|l|}{ P value } & 0.000 & 0.000 & 0.000 & 0.000 & 0.000 & 0.000 \\
\hline \multicolumn{9}{|l|}{ Breed effect } \\
\hline \multicolumn{3}{|l|}{ Aseel } & $51.68^{\mathrm{a}} \pm 1.61$ & $49.27^{\mathrm{a}} \pm 1.40$ & $60.25^{\mathrm{a}} \pm 1.49$ & $64.19^{\mathrm{a}} \pm 1.84$ & $52.48^{\mathrm{a}} \pm 1.46$ & $73.97^{\mathrm{a}} \pm 2.46$ \\
\hline \multicolumn{3}{|l|}{ Kadaknath } & $36.03^{\mathrm{b}} \pm 1.25$ & $22.27^{\mathrm{b}} \pm 1.15$ & $30.64^{\mathrm{b}} \pm 1.23$ & $38.69^{\mathrm{b}} \pm 1.26$ & $39.37^{\mathrm{b}} \pm 1.35$ & $47.51^{b} \pm 1.52$ \\
\hline \multicolumn{3}{|l|}{ P-value } & 0.000 & 0.000 & 0.000 & 0.000 & 0.000 & 0.000 \\
\hline
\end{tabular}


Table.6 Influence of breed on feed intake per head $(\mathrm{g})$ during brooder phase

\begin{tabular}{|c|c|c|c|c|c|c|c|}
\hline Breeds & Sex & 1 week & 2 week & 3 week & 4 week & 5 week & 6 week \\
\hline \multicolumn{8}{|c|}{ Interaction effect } \\
\hline \multirow[t]{2}{*}{ Aseel } & Females & $56.88^{\mathrm{a}} \pm 1.97$ & $84.73^{b} \pm 3.58$ & $106.37^{\mathrm{b}} \pm 5.51$ & $142.08^{\mathrm{a}} \pm 10.22$ & $158.78^{b} \pm 7.60$ & $212.24^{b} \pm 9.78$ \\
\hline & Males & $49.30^{\mathrm{b}} \pm 1.27$ & $96.76^{\mathrm{a}} \pm 0.88$ & $117.78^{\mathrm{a}} \pm 5.66$ & $123.90^{\mathrm{b}} \pm 2.74$ & $197.07^{\mathrm{a}} \pm 1.03$ & $226.00^{\mathrm{a}} \pm 2.31$ \\
\hline \multirow[t]{2}{*}{ Kadaknath } & Females & $34.33^{c} \pm 1.66$ & $56.83^{c} \pm 1.67$ & $80.51^{c} \pm 1.43$ & $96.35^{c} \pm 1.32$ & $103.63^{c} \pm 2.10$ & $124.04^{c} \pm 6.09$ \\
\hline & Males & $35.95^{\mathrm{c}} \pm 1.14$ & $51.56^{c} \pm 2.38$ & $70.82^{c} \pm 2.03$ & $92.81^{\mathrm{c}} \pm 2.14$ & $100.45^{\mathrm{c}} \pm 1.68$ & $136.23^{\mathrm{c}} \pm 4.45$ \\
\hline \multicolumn{2}{|l|}{ P-value } & 0.000 & 0.000 & 0.000 & 0.000 & 0.000 & 0.000 \\
\hline \multicolumn{8}{|l|}{ Breed effect } \\
\hline \multicolumn{2}{|l|}{ Aseel } & $53.09^{\mathrm{a}} \pm 1.99$ & $90.75^{\mathrm{a}} \pm 3.16$ & $112.08^{\mathrm{a}} \pm 4.36$ & $132.99^{\mathrm{a}} \pm 6.24$ & $177.92^{\mathrm{a}} \pm 9.22$ & $219.12^{\mathrm{a}} \pm 5.45$ \\
\hline \multicolumn{2}{|l|}{ Kadaknath } & $35.14^{\mathrm{b}} \pm 0.97$ & $54.20^{\mathrm{b}} \pm 1.76$ & $75.67^{b} \pm 2.43$ & $94.58^{\mathrm{b}} \pm 1.37$ & $102.04^{b} \pm 1.40$ & $130.13^{\mathrm{b}} \pm 4.33$ \\
\hline \multicolumn{2}{|l|}{ P-value } & 0.000 & 0.000 & 0.000 & 0.000 & 0.000 & 0.000 \\
\hline
\end{tabular}

Means within columns bearing different superscripts differ significantly $(\mathrm{p}<0.05)$.

Table.7 Influence of breed on feed intake per head $(\mathrm{g})$ during grower phase

\begin{tabular}{|c|c|c|c|c|c|c|c|c|}
\hline Breed & Sex & Rearing & 7 week & 8 week & 9 week & 10 week & 11 week & 12 week \\
\hline \multicolumn{9}{|c|}{ Interaction effect } \\
\hline \multirow[t]{4}{*}{ Aseel } & \multirow[t]{2}{*}{ Females } & Cage & $357.41^{\mathrm{ab}} \pm 18.53$ & $486.24^{\mathrm{a}} \pm 1.71$ & $381.56^{\mathrm{b}} \pm 34.83$ & $460.87^{\mathrm{a}} \pm 7.48$ & $491.52^{\mathrm{a}} \pm 5.11$ & $550.94^{\mathrm{a}} \pm 18.54$ \\
\hline & & Litter & $408.3^{\mathrm{a}} \pm 38.4$ & $376.3^{\mathrm{b}} \pm 22.5$ & $328.0^{\mathrm{bc}} \pm 10.1$ & $313.5^{\mathrm{c}} \pm 8.8$ & $480.7^{\mathrm{a}} \pm 39.9$ & $543.9^{\mathrm{ab}} \pm 10.8$ \\
\hline & \multirow[t]{2}{*}{ Males } & Cage & $349.08^{\mathrm{b}} \pm 0.94$ & $386.28^{\mathrm{b}} \pm 51.40$ & $469.85^{\mathrm{a}} \pm 6.57$ & $478.43^{\mathrm{a}} \pm 12.61$ & $487.03^{\mathrm{a}} \pm 18.57$ & $529.87^{\mathrm{ab}} \pm 8.53$ \\
\hline & & Litter & $337.9^{\mathrm{b}} \pm 15.4$ & $275.6^{c} \pm 12.4$ & $285.0^{\mathrm{c}} \pm 37.7$ & $327.5^{b c} \pm 40.5$ & $396.5^{c} \pm 16.4$ & $392.7^{c} \pm 24.6$ \\
\hline \multirow[t]{4}{*}{ Kadaknath } & \multirow[t]{2}{*}{ Females } & Cage & $125.55^{\mathrm{c}} \pm 3.86$ & $158.82^{\mathrm{d}} \pm 8.99$ & $330.61^{b c} \pm 5.68$ & $376.47^{\mathrm{b}} \pm 6.05$ & $434.00^{\mathrm{ab}} \pm 23.71$ & $553.29^{\mathrm{a}} \pm 30.08$ \\
\hline & & Litter & $162.7^{\mathrm{c}} \pm 7.9$ & $155.5^{\mathrm{d}} \pm 15.7$ & $180.6^{\mathrm{d}} \pm 13.2$ & $242.0^{\mathrm{d}} \pm 15.4$ & $294.5^{\mathrm{d}} \pm 17.3$ & $314.2^{\mathrm{d}} \pm 26.7$ \\
\hline & \multirow[t]{2}{*}{ Males } & Cage & $179.29^{c} \pm 10.37$ & $218.89^{\mathrm{cd}} \pm 13.26$ & $344.82^{b c} \pm 21.96$ & $396.42^{b} \pm 4.96$ & $405.24^{c} \pm 6.52$ & $567.0^{6 c} \pm 11.43$ \\
\hline & & Litter & $147.9^{\mathrm{c}} \pm 8.7$ & $154.8^{\mathrm{d}} \pm 6.0$ & $193.7^{\mathrm{d}} \pm 6.3$ & $274.0^{\mathrm{cd}} \pm 13.2$ & $331.0^{\mathrm{d}} \pm 29.5$ & $496.0^{\mathrm{bc}} \pm 30.6$ \\
\hline P value & & & 0.000 & 0.000 & 0.000 & 0.000 & 0.000 & 0.000 \\
\hline \multicolumn{9}{|l|}{ Breed effect } \\
\hline \multicolumn{3}{|l|}{ Aseel } & $363.19^{\mathrm{a}} \pm 12.64$ & $381.10^{\mathrm{a}} \pm 25.61$ & $366.10^{\mathrm{a}} \pm 23.65$ & $395.08^{\mathrm{a}} \pm 24.47$ & $463.92^{\mathrm{a}} \pm 15.52$ & $504.35 \pm 20.86$ \\
\hline \multicolumn{3}{|l|}{ Kadaknath } & $153.86^{\mathrm{b}} \pm 6.88$ & $172.00^{\mathrm{b}} \pm 9.56$ & $262.43^{b} \pm 23.51$ & $322.24^{\mathrm{b}} \pm 20.31$ & $366.18^{\mathrm{b}} \pm 19.10$ & $482.65 \pm 32.34$ \\
\hline \multicolumn{3}{|l|}{ P-value } & 0.000 & 0.000 & 0.005 & 0.032 & 0.001 & 0.0578 \\
\hline
\end{tabular}

Means within columns bearing different superscripts differ significantly $(\mathrm{p}<0.05)$. 
Table.8 Influence of breed on feed efficiency during brooder phase

\begin{tabular}{|c|c|c|c|c|c|c|c|}
\hline Breeds & Sex & 1 week & 2 week & 3 week & 4 week & 5 week & 6 week \\
\hline \multicolumn{8}{|c|}{ Interaction effect } \\
\hline \multirow[t]{2}{*}{ Aseel } & Females & $3.69^{\mathrm{a}} \pm 0.08$ & $3.53^{\mathrm{a}} \pm 0.16$ & $3.35^{\mathrm{a}} \pm 0.18$ & $3.67^{\mathrm{a}} \pm 0.33$ & $3.05 \pm 0.16$ & $2.95 \pm 0.23$ \\
\hline & Males & $3.26^{\mathrm{a}} \pm 0.15$ & $2.66^{\mathrm{c}} \pm 0.05$ & $3.42^{\mathrm{a}} \pm 0.18$ & $3.57^{\mathrm{a}} \pm 0.20$ & $3.57 \pm 0.03$ & $3.54 \pm 0.15$ \\
\hline \multirow[t]{2}{*}{ Kadaknath } & Females & $2.26^{\mathrm{b}} \pm 0.19$ & $3.13^{\mathrm{b}} \pm 0.11$ & $2.89^{\mathrm{b}} \pm 0.08$ & $2.99^{\mathrm{b}} \pm 0.15$ & $3.01 \pm 0.21$ & $3.08 \pm 0.33$ \\
\hline & Males & $2.61^{b} \pm 0.09$ & $2.70^{c} \pm 0.06$ & $2.79^{b} \pm 0.05$ & $3.36^{\mathrm{a}} \pm 0.11$ & $3.20 \pm 0.17$ & $3.01 \pm 0.08$ \\
\hline \multicolumn{2}{|l|}{ P-value } & 0.000 & 0.000 & 0.001 & 0.025 & 0.196 & 0.123 \\
\hline \multicolumn{8}{|l|}{ Breed effect } \\
\hline \multicolumn{2}{|l|}{ Aseel } & $3.47^{\mathrm{a}} \pm 0.12$ & $3.09^{\mathrm{a}} \pm 0.21$ & $3.38^{\mathrm{a}} \pm 0.12$ & $3.62^{\mathrm{a}} \pm 0.17$ & $3.31 \pm 0.14$ & $3.24 \pm 0.18$ \\
\hline \multicolumn{2}{|l|}{ Kadaknath } & $2.44^{\mathrm{b}} \pm 0.12$ & $2.91^{\mathrm{b}} \pm 0.11$ & $2.84^{\mathrm{b}} \pm 0.05$ & $3.18^{\mathrm{b}} \pm 0.12$ & $3.11 \pm 0.13$ & $3.05 \pm 0.15$ \\
\hline \multicolumn{2}{|l|}{ P-value } & 0.000 & 0.468 & 0.001 & 0.059 & 0.308 & 0.432 \\
\hline
\end{tabular}

Means within columns bearing different superscripts differ significantly $(\mathrm{p}<0.05)$.

Table.9 Influence of breed on feed efficiency during grower phase

\begin{tabular}{|c|c|c|c|c|c|c|c|c|}
\hline Breed & Sex & Age & 7 week & 8 week & 9 week & 10 week & 11 week & 12 week \\
\hline \multicolumn{9}{|c|}{ Interaction effect } \\
\hline \multirow[t]{4}{*}{ Aseel } & \multirow[t]{2}{*}{ Females } & Cage & $3.80^{\mathrm{de}} \pm 0.38$ & $7.68 \pm 0.49$ & $5.36^{\mathrm{d}} \pm 0.93$ & $5.91^{\mathrm{b}} \pm 0.44$ & $7.81^{\mathrm{b}} \pm 0.42$ & $7.23^{\mathrm{b}} \pm 1.03$ \\
\hline & & Litter & $5.46^{\mathrm{abcd}} \pm 1.24$ & $8.06 \pm 0.57$ & $6.03^{b c} \pm 0.28$ & $5.84^{\mathrm{b}} \pm 0.44$ & $8.76^{\mathrm{b}} \pm 0.40$ & $7.87^{\mathrm{ab}} \pm 0.28$ \\
\hline & \multirow[t]{2}{*}{ Males } & Cage & $4.29^{\mathrm{cde}} \pm 0.12$ & $7.86 \pm 0.89$ & $7.65^{\mathrm{abc}} \pm 0.48$ & $6.20^{\mathrm{b}} \pm 0.05$ & $9.01^{\mathrm{ab}} \pm 0.47$ & $5.05^{c} \pm 0.49$ \\
\hline & & Litter & $3.57^{\mathrm{e}} \pm 0.30$ & $6.66 \pm 0.25$ & $5.30^{\mathrm{d}} \pm 0.54$ & $5.91^{\mathrm{b}} \pm 0.59$ & $8.73^{b} \pm 0.03$ & $7.32^{b} \pm 0.82$ \\
\hline \multirow[t]{4}{*}{ Kadaknath } & \multirow[t]{2}{*}{ Females } & Cage & $5.97^{\mathrm{abc}} \pm 0.49$ & $7.01 \pm 0.16$ & $9.09^{\mathrm{a}} \pm 0.09$ & $7.90^{\mathrm{ab}} \pm 0.31$ & $8.78^{b} \pm 0.29$ & $9.66^{\mathrm{a}} \pm 0.13$ \\
\hline & & Litter & $7.17^{\mathrm{a}} \pm 0.29$ & $7.49 \pm 0.24$ & $8.43^{\mathrm{ab}} \pm 0.59$ & $7.16^{\mathrm{b}} \pm 0.59$ & $9.06^{\mathrm{ab}} \pm 0.42$ & $8.10^{\mathrm{ab}} \pm 0.33$ \\
\hline & \multirow[t]{2}{*}{ Males } & Cage & $6.15^{\mathrm{ab}} \pm 0.26$ & $8.93 \pm 1.21$ & $8.07^{\mathrm{ab}} \pm 1.03$ & $9.33^{\mathrm{a}} \pm 1.08$ & $8.04^{\mathrm{b}} \pm 0.94$ & $8.88^{\mathrm{ab}} \pm 0.91$ \\
\hline & & Litter & $4.95^{\text {bcde }} \pm 0.50$ & $7.18 \pm 1.05$ & $7.24^{\mathrm{abc}} \pm 1.22$ & $7.89^{\mathrm{ab}} \pm 1.07$ & $10.51^{\mathrm{a}} \pm 0.45$ & $8.53^{\mathrm{ab}} \pm 0.21$ \\
\hline \multicolumn{3}{|l|}{ P value } & 0.003 & 0.481 & 0.013 & 0.014 & 0.046 & 0.004 \\
\hline \multicolumn{9}{|l|}{ Breed effect } \\
\hline \multicolumn{3}{|l|}{ Aseel } & $4.28 \pm 0.36$ & $7.56 \pm 0.30$ & $6.08^{\mathrm{b}} \pm 0.38$ & $5.96^{\mathrm{b}} \pm 0.19$ & $8.58^{\mathrm{b}} \pm 0.21$ & $6.87^{\mathrm{b}} \pm 0.44$ \\
\hline \multicolumn{3}{|l|}{ Kadaknath } & $6.06 \pm 0.29$ & $7.65 \pm 0.41$ & $8.21^{\mathrm{a}} \pm 0.41$ & $8.01^{\mathrm{a}} \pm 0.42$ & $9.09^{\mathrm{a}} \pm 0.37$ & $8.80^{\mathrm{a}} \pm 0.27$ \\
\hline \multicolumn{3}{|l|}{ P-value } & 0.052 & 0.560 & 0.000 & 0.000 & 0.037 & 0.012 \\
\hline
\end{tabular}

Means within columns bearing different superscripts differ significantly $(\mathrm{p}<0.05)$. 
Feed intake in Kadaknath for 4,8 and $12^{\text {th }}$ week was higher than feed intake recorded by Parmar (2003) who reported 4,8 and $12^{\text {th }}$ week feed intake as $69.25 \mathrm{~g}, 133.63 \mathrm{~g}, 290.50$ $\mathrm{g}$ respectively. Caged birds had higher feed intake than birds on deep litter. Haunshi et al., (2009) reported $4^{\text {th }}$ week feed intake of Miri, Gramapriya and Vanaraja as $62.33 \mathrm{~g}, 259.36 \mathrm{~g}$ and $225.07 \mathrm{~g} .4^{\text {th }}$ week feed intake of Aseel and Kadaknath was 132.99 \pm 6.24 and $94.58 \pm 1.37 \mathrm{~g}$ which is higher than feed intake of Miri but lower than feed intake of Gramapriya and Vanaraja reported by Haunshi et al., (2009).

\section{Feed Conversion Ratio (FCR)}

FCR is an important indicator of production performance. Constant efforts by scientists have successfully brought down the FCR for broilers. Statistical analysis shows significant $(\mathrm{P}<0.05)$ difference exists between groups (Table 8 and 9). Kundu et al., (2015) reported FCR of Vanaraja, White Nicobari, Black Nicobari and Brown Nicobari as 2.6, 3.6, 3.9 and 4.17 respectively. $4^{\text {th }}$ week FCR for Aseel male, Aseel female, Kadaknath male and Kadaknath female were $3.57 \pm 0.20,3.67 \pm 0.33$, $3.36 \pm 0.11$ and $2.99 \pm 0.15$ respectively. Breed effect on FCR was highly significant $(\mathrm{P}<0.01)$ for $1,2,9$ and 10 weeks. $4^{\text {th }}$ week FCR for Aseel and Kadaknath breeds was 3.62 \pm 0.17 and $3.18 \pm 0.1$ which is less than findings of Das et al., (2016) for CARI Sonali with FCR of $4.04 \pm 0.07$. FCR for Kadaknath is in close agreement to the observations of Parmar (2003). Male birds had higher FCR for 4, 5, 6, 9, 10 and 11 weeks. FCR increased with age which can be explained by slowing of growth rate with age. Tadelle et al., (2003) reported that females of Horro and Chefe ecotypes had FCR as 4.4 and 5.8 and males had 5.5 and 5.0 at 6-12 weeks respectively.

The Aseel birds (both males and females) performed better than the Kadaknath (males and females) in terms of growth rate, feed efficiency during hot-humid conditions. Hence, Aseel birds may be used for crossbreeding purpose for the development of rural varieties.

\section{References}

Arora, G., Mishra, S. K., Nautiyal, B., Pratap, S. O., Gupta, A., Beura, C. K and Singh, D. P. (2011). Genetics of hyperpigmentation associated with the Fibromelanosis gene (Fm) and analysis of growth and meat quality traits in crosses of native Indian Kadaknath chickens and non-indigenous breeds. British Poultry Science, 52(6): 675-685.

Biswas, A., Mohan, J and Sastry, K. V. H. 2010. Effect of vitamin $\mathrm{E}$ on production performance and egg quality traits in Indian Native Kadaknath hen. AsianAustralian Journal of Animal Science, 23: 396-400.

Chatterjee, R. N., Sharma, R. P., Reddy, M. R., Niranjan, $M$ and Reddy, B. L. N. 2007. Growth, body conformation and immune responsiveness in two Indian native chicken breeds. Livestock Research for Rural Development, 19: 151.

Crawford, R. D and Christman, C. J. 1992. Heritage hatchery networks in poultry conservation.

In: Genetic conservation of domestic livestock, $\mathrm{CAB}$ International, Oxford, UK. Pp. 212-222.

Das, A.K., Kumar, S., Mishra, A. K., Rahim, A and Kokate, L. S. 2016. Evaluating body conformation and feed efficiency characteristics in CARI-Sonali grower chicken. The Indian Journal of Animal Sciences, 86(2): 192-196.

Gupta, R. K., Singh, M., Singh, U and Gurung, B. S. 2000. Feed efficiency and carcass characteristics of Aseel chicken. The Indian Journal of Animal Sciences, 70(11): 1170-1171.

Haunshi, S., Doley, S., Shakuntala, I and Bujarbaruah, K. M. 2009. Production performance of indigenous chicken of north-eastern region and improved varieties developed for backyard farming. The Indian Journal of Animal Sciences, 79(9): 901. 
Haunshi, S., Niranjan, M., Shanmugam, M., Padhi, M. K., Reddy, M. R., Sunitha, R., Rajkumar, $\mathrm{U}$ and Panda, A. K. 2011.Characterization of two Indian native chicken breeds for production, egg and semen quality and welfare traits. Poultry Science, 90: 314-320.

Kundu, A., De, A. K., Kundu, M. S., Sunder, J., Jeyakumar, S and Sujatha, T. 2015. Production performance of indigenous Nicobari fowls, Vanaraja and their various F1 crosses under hot and humid climate of Andaman and Nicobar Islands, India. The Indian Journal of Animal Science, 85(2): 172-177.

Mashaly, M. M., Hendricks, G. L., Kalama, M. A., Gehad, A. E., Abbas, A. O and Patterson, P H. 2004. Effect of heat stress on production parameters and immune responses of commercial laying hens. Poultry Science, 83(6): 889-894.

Mohan, J., Sastry, K. V. H., Moudgal, R. P and Tyagi, J. S. 2008. Production and other characteristics of Aseel Peela desi hens under normal rearing system. Indian Journal of Poultry Science, 43: 217-219.

Panda, B and Mahapatra, S. C. 1989.Common breeds of poultry. In: Poultry Production. ICAR, New Delhi, India, Pp. 6-18.

Panda, B and Praharaj, N. K. 2002. Conservation of indigenous chicken germplasm in India: Past, present and future scenario. Proceedings of National Workshop on characterization and conservation of indigenous poultry germplasm, 26-27 February 2002, CARI, Port Blair, Andaman, India. Pp. 17-27.

Parmar, S. N. S., Shrivastava, P. N., Tomar, S. S., Pillai, P. V. A and Tomar, I. S. 2003. Characterization of Kadaknath breed of poultry. JNKVV, Technical Bulletin, DRS/2003/01.

\section{How to cite this article:}

Shanmathy, M., J.S. Tyagi, M. Gopi, J. Mohan, P. Beulah and Ravi Kumar, D. 2018. Comparative Assessment on Performance of Aseel and Kadaknath in Hot and Humid Conditions in Tropics. Int.J.Curr.Microbiol.App.Sci. 7(05): 2156-2165. doi: https://doi.org/10.20546/ijcmas.2018.705.251
Pratap, S. O., Mishra, S. K., Khan, A. A and Singh, D. P. 2014. Comparative Growth Performance of Indigenous Kadaknath and Exotic White Leghorn Chicken under Intensive Management. SKUAST Journal of Research, 16(2): 1

Rao, G. V and Thomas, P. C. 1984. The breed characteristics of Kadaknath breed of indigenous (Desi) chicken; Avian Research, 68: 55-57.

Siegel, H. S. 1995. Stress, strains and resistance. British Poultry Science, 36(1): 3-22.

Tadelle, D., Kijora, C and Peters, K. J. 2003. Indigenous chicken ecotypes in Ethiopia: growth and feed utilization potentials. International Journal of Poultry Science, 2(2): 144-152.

Thakur, M. S., Parmar, S. N. S and Pillai, P. V. A. 2006. Studies on growth performance in Kadaknath breed of poultry. Livestock Research for Rural Development, 18: 116.

Valavan, S., Ezhil A. V., Omprakash, A., Bharatidhasan (??) and Saravana Kumar, R. 2016. Production performance of Aseel under Indian tropical condition. International Journal of applied Pure Science and Agriculture, 2(11): 107-110.

Vecerek, V., Strakova, E., Suchy, $\mathrm{P}$ and Voslarova, E. 2002. Influence of high environmental temperature on production and haematological and biochemical indexes in broiler chickens. Czech Journal of Animal Science, 47(5): 176-182.

Zulkifli, I., Abdullah, N., Azrin, N and MandHo, Y. W. 2000. Growth performance and immune response of two commercial broiler strains fed diets containing Lactobacillus cultures and oxytetracycline under heat stress conditions. British Poultry Science, 41(5): 593-59. 